\title{
Altruismus, Familie, Religion: Testamente um 1800
}

Am 7. Februar 1734 starb in Linz einer der prominenteren Bürger der Stadt, der Handelsmann, Stadtrichter und Bürgermeister Johann Adam Pruner, im Alter von 62 Jahren. Pruner (Bruder des drei Jahre älteren Baumeisters Johann Michael Pruner) hinterließ bei seinem Tod ein beträchtliches Vermögen, wovon er einigen Verwandten teils größere Legate zudachte; seine Ehefrau war bereits früher verstorben, Kinder hatte er nicht, Universalerbin war seine Schwester. Wie üblich, bedachte Pruner verschiedene fromme Bruderschaften mit Legaten, bestellte etliche Seelenmessen für sich und vergaß auch die örtlichen Spitäler und Siechenhäuser sowie einige Gotteshäuser nicht; 1700 Gulden sollten angelegt werden, um für 27 arme Kinder aus Linz das jährliche Schulgeld zu bezahlen. Das umfangreichste Vermächtnis erhielten aber andere: Eine neu einzurichtende Stiftung sollte für 27 Kinder und für 54 erwachsene Arme sorgen, wofür Pruner jeweils 54.000 Gulden widmete. Für diesen Zweck sollte auch ein Stiftungsgebäude errichtet werden (das heute noch bestehende Prunerstift), das mit einem Gotteshaus (das heute als Altkatholische Kirche dient) und einem Benefiziaten zu versehen war; als Aufwand für das Gebäude waren 36.000 Gulden vorgesehen, für die Kirche weitere 22.000 Gulden. Das Benefizium war mit 12.000 Gulden zu dotieren. Der Benefiziat hatte eine Messe pro Woche frei, alle anderen Messen mussten für den Stifter gelesen werden. ${ }^{1}$

${ }^{1}$ OÖLA, LGA 106 (Stadtrecht), 105.
Das Testament Adam Pruners zeigt in extremer Ausformung Eigenarten barocker Testamente und ihren Zusammenhang mit den vielen Aspekten von Frömmigkeit, Mildtätigkeit, Sorge um Angehörige, Statusbewusstsein und spezifischen wirtschaftlichen Möglichkeiten von Testatoren. Manche dieser Eigenarten blieben auch in der zweiten Hälfte des 18. Jahrhunderts in den Grundzügen bestehen, wandelten sich aber in ihrer konkreten Ausformung, anderes verschwand überhaupt. Im Mittelpunkt dieser Überlegungen soll aber nicht so sehr die Bedeutung der österreichischen Aufklärung für den Wandel in der Religiosität stehen, sondern die Frage nach den Motiven von Testatoren jener Zeit, nach dem Nutzen von testamentarischen Bestimmungen für die Testatoren selbst, für ihre Familie und für Außenstehende.

So einprägsam das Testament des Johann Adam Pruner auch ist, es ist für seine Zeit insofern atypisch, als es der Letzte Wille eines reichen Mannes ist. Testamente gehören nämlich zu den wenigen Quellensorten, in denen auch ganz gewöhnliche Personen Äußerungen über sich selbst und ihre Sicht der Welt abgeben. "Gewöhnliche Personen“, das sind Personen, die kein hohes Einkommen und kein großes Vermögen haben müssen. Tatsächlich verfügen wir seit Jahrhunderten über Testamente von Menschen aller Einkommensklassen, Berufe, jeden Ausbildungsstands und beiderlei Geschlechts. ${ }^{2}$

\footnotetext{
${ }^{2} \mathrm{Zu}$ Testamenten als Quelle PAMMER, Testamente und Verlassenschaftsabhandlungen.
} 
Dass es sich bei den Testamenten um eigene Äußerungen der Testatoren handelt, stimmt jedenfalls in dem Sinn, dass testamentarische Verfügungen als letzter Wille des Erblassers anerkannt werden, wenn die Formvorschriften erfüllt sind. Dies bedeutet klarerweise nicht, dass ein Testator gehandelt haben muss, ohne dabei von anderen beeinflusst worden zu sein. Einflüsse auf letztwillige Erklärungen mögen von juristischen Beratern kommen, die etwa einen Testator auf die Bahn der Konvention lenken, aber auch von Familienangehörigen oder Seelsorgern, die spezifische Interessen verfolgen. Die genauen Abläufe liegen im Dunkeln, doch regte das Thema zu Phantasien an. So wurde in einer theresianischen Verordnung aus dem Jahr 1771 verboten, dass Priester für andere Personen das Testament niederschrieben; Testamente, die Priester für andere niedergeschrieben hatten, wurden dadurch ungültig. Auch als Testamentszeugen waren Priester nicht zugelassen. Die Begründung lautete:

„Da Uns nun glaubwürdig beygebracht worden, dass hin und wieder auf dem Lande bey Absterben der Bauern, und zuweilen auch in Städten bey wohlhabigen Burgersleuten, die zum letzten Beystande für den Kranken herbeygeholten Geistlichen sich zur Verfertigung der Testamente gebrauchen lassen, und solchen Falles, hauptsächlich, wenn nachmals wie es nicht selten geschiehet, in Absicht auf das ruckgebliebene Vermögen, grosse, die Erben beschwerende fromme Vermächtnisse, Stiftungen oder Legaten für das Kloster desjenigen Geistlichen, der das Testament verfasset hat, herauskommen, viele Klagen deshalben wider die Geistlichkeit hervorbrechen. ${ }^{\text {"3 }}$

Auch §591 des Allgemeinen Bürgerlichen Gesetzbuchs von 1811 (aufgehoben 1914) legte fest,

\footnotetext{
${ }^{3}$ Verordnung vom 4. 9. 1771, Sammlung der Kaiserlich=Königlichen Landesfürstlichen Gesetze und Verordnungen 41-42.
}

dass Mitglieder geistlicher Orden nicht als Zeugen bei Testamenten fungieren dürften.

Nun waren schon vor Erlass dieser Bestimmung Geistliche an der Errichtung von Testamenten tatsächlich kaum als Schreiber oder Zeugen beteiligt. Testatoren, die bereits todkrank und nicht mehr zum Schreiben in der Lage waren, ließen sich bei der Errichtung ihres Letzten Willens gewöhnlich von Anwälten oder Notaren und nicht von Priestern helfen. Ohnehin wurden aber viele Testamente geraume Zeit vor dem Tod und oft genug von den Erblassern eigenhändig geschrieben; die frommen Vermächtnisse waren in solchen Testamenten sogar eher umfangreicher als in den kurz vor dem Ableben geschriebenen Dokumenten.

Dennoch bedeutet dies nicht, dass diese Erblasser handelten, ohne dabei von anderen und eben auch von Geistlichen beeinflusst worden $\mathrm{zu}$ sein. Diese Einflussnahme hat man sich freilich nicht so vorzustellen, wie es offenbar die theresianischen Beamten taten - Geistlicher nützt die Angst des Todkranken aus, um diesem fromme Legate einzureden oder sogar aus eigenem solche Legate in das Testament hineinzuschreiben -, sondern die Einflussnahme erfolgte weniger direkt: Es bestand ohnehin die Konvention, in letztwilligen Erklärungen fromme Legate vorzusehen, und diese Konvention wurde sicherlich auch dadurch gestützt, dass die Seelsorger den Gläubigen immer wieder die guten Gründe für solche Legate in Erinnerung riefen.

\section{Fromme Legate zwischen Eigeninteresse und Altruismus}

Vorkommen und Umfang frommer Legate sind Merkmale, die Testamente des 18. Jahrhunderts von jenen des 19. und 20. Jahrhunderts deutlich unterscheiden. Die Besonderheit dieser Legate liegt in ihrer Zweideutigkeit: Sie sind in dem Sinn altruistisch, dass sie Menschen zugutekommen, mit denen die Erblasser wenig oder 
nichts verbindet und die der Erblasser persönlich oft gar nicht kennt. Andererseits sollen sie doch auch dem Erblasser einen Nutzen bringen. Beides - das altruistische und das egoistische Element - bedarf einer Erläuterung. Was ist der Zweck eines frommen Legats?

Insoweit ein Legat aus Frömmigkeit gemacht wird, liegt die Motivation jedenfalls in einem religiösen Anliegen: Der Testator macht das Legat, um sein eigenes Seelenheil oder das Seelenheil einer anderen Person zu befördern. Diese Förderung des Seelenheils kann durch ein Werk der Nächstenliebe oder durch andere Akte erfolgen. Der Zusammenhang zwischen frommen Akten und dem Seelenheil war in der Neuzeit in der katholischen Kirche eine längst akzeptierte Wahrheit. Thomas von Aquin betrachtete Messen, Gebete und Almosen als die drei wirksamsten Fürbitten für die Verstorbenen: Die Messe, das heißt hier die Seelenmesse, ist die stärkste Fürbitte, weil mit der Messe und dem Messopfer Christus selbst zum Fürsprecher gemacht wird; mit Gebeten werden Gott und die Heiligen um Hilfe angerufen; die Wirksamkeit der Almosen kam aus ihrem Ursprung in der Nächstenliebe, einem der beiden wichtigsten Gebote, und war daher aus sich heraus gegeben (darüber hinaus war das Almosen oft auch mit Gebeten des Almosenempfängers für seinen Wohltäter verbunden). ${ }^{4}$ Alle diese Akte erfolgten in der Form, dass Testatoren in ihren Testamenten Legate für eine bestimmte Zahl von (einmalig oder periodisch zu wiederholenden) Seelenmessen und bestimmte karitative Werke vorsahen und allenfalls forderten, dass von diesen oder jenen Empfängern für sie gebetet werden sollte. Mittels der Messen, Gebete und mildtätigen Werke wurde so auch ein Bezug zwischen Lebenden und Verstorbenen hergestellt. Diese Auffassung blieb über Jahrhunderte bestehen und war im Barockkatholizismus fest etabliert. Dies hatte zur Folge,

${ }^{4}$ LE GoFF, Geburt des Fegefeuers 334. dass noch um die Mitte des 18. Jahrhunderts ein beträchtlicher Teil der katholischen Bevölkerung in den erläuternden Bemerkungen ihrer Testamente mildtätige Werke ausdrücklich in Verbindung mit dem eigenen Seelenheil brachte. $\mathrm{Ob}$ diese Bekundungen über die rhetorische Funktion hinaus auch Ausdruck einer verinnerlichten religiösen Haltung waren, lässt sich freilich nicht entscheiden. Es ist anzunehmen, dass ein Teil der Testatoren fromme Legate bloß aus Gründen der Konvention machte und die transzendente Begründung nur rhetorischen Charakter hatte.

Nimmt man die religiöse Begründung ernst, ist das fromme Legat also eine eigennützige Angelegenheit. Der altruistische Aspekt ist je nachdem mehr oder weniger wichtig: Die Abhaltung von Seelenmessen war zwar eine Einkommensquelle für zahlreiche Priester bzw. auch die Klöster, denen sie angehörten, doch wird man das häufige Vorkommen entsprechender Legate in Testamenten des 18. Jahrhunderts normalerweise nicht mit dem Wunsch der Erblasser erklären können, die Priester zu unterstützen. In einem solchen Fall wäre es ja auch möglich gewesen, einfach ein sonstiges Vermächtnis (ohne die Verpflichtung, Messen zu lesen) vorzusehen, was mindestens in Form von Legaten für Gotteshäuser (weniger für die Priester persönlich) öfters vorkam; auch der über Jahrzehnte feste Preis pro Seelenmesse (ein halber Gulden für die einzelne Messe, fünfzig Gulden für einen Jahrtag, das ist eine auf ewig abzuhaltende jährliche Messe) deutet auf eine geschäftsmäßige Sicht der Dinge hin. Selten findet man den Wunsch, die vom Erblasser gewünschten Seelenmessen von bedürftigen Geistlichen halten $\mathrm{zu}$ lassen, ${ }^{5}$ und ausnahmsweise wird angeordnet, die Seelenmessen seien, so die eigene Seele ihrer nicht

\footnotetext{
${ }^{5}$ Etwa OÖLA, LGA 33 (Landrecht), 105; LGA 41 (Landrecht), 543; LGA 80 (Stadtrecht), 800.
} 
mehr bedürfe, zum Nutzen anderer armer Seelen anzurechnen. ${ }^{6}$

Hingegen stand im Bereich der karitativen Legate der altruistische Aspekt eher im Vordergrund. Eine Ausnahme sind die Legate für Arme, die am Begräbnis teilnehmen und für den Verstorbenen beten sollten, wofür ihnen ein kleiner Geldbetrag zugedacht war; dieses Legat nützte somit beiden Seiten. Bei den sonstigen, quantitativ weit wichtigeren karitativen Vermächtnissen wurde kaum von Gegenleistungen der Legatare, etwa in Form von Gebeten für den Erblasser, gesprochen, sondern man bestimmte in der Regel bloß einen Geldbetrag für Spitäler, Hausarme und so weiter. Freilich gab es davon abweichende Einzelfälle wie den eingangs beschriebenen Adam Pruner, der seine mildtätige Stiftung mit einem umfangreichen Programm von Seelenmessen verband; und sicherlich beteten Spitalsinsassen immer wieder allgemein für die Wohltäter des Spitals.

In den meisten Fällen wäre es jedenfalls dem Wortlaut der Testamente nach ohne weiteres möglich, dass Testatoren mit ihren karitativen Klauseln einfach ein soziales Anliegen verfolgten, also den Wunsch, Armen zu helfen oder Bildung zu fördern, wie er auch in einem völlig säkularisierten Kontext vorkommt. Im Einzelfall ist die Motivlage oft nicht eindeutig festzustellen. Es ist durchaus denkbar, dass in einem Testament Vermächtnisse für Arme aus religiöser Motivation und mit der Hoffnung auf einen eigenen Nutzen (in Form von Gebeten, die Spitalspfründner für ihre Wohltäter verrichteten, oder sonst eine milde Behandlung im Jenseits) gemacht wurden, ohne dass der Text irgendeinen religiösen Bezug erkennen lässt.

Ein weiteres eigennütziges Motiv von frommen Testatoren liegt in der repräsentativen Funktion von Frömmigkeit und Mildtätigkeit. Die Einzel-

\footnotetext{
${ }^{6}$ Etwa OÖLA, LGA 32 (Landrecht), 21; LGA 109 (Stadtrecht), 988.
}

heiten folgen zeitspezifischen Gegebenheiten, weil Repräsentation und Dokumentation von Status je nach zeitgenössischem Kontext auf unterschiedliche Weise erfolgen. Schon seit dem späten 18. Jahrhundert war etwa die Abhaltung von täglichen Seelenmessen kaum mehr geeignet, den besonderen Rang der betreffenden Person zu unterstreichen; auch die Verteilung von Handalmosen bei Begräbnissen würde heute eine solche Funktion nicht erfüllen. Anders sieht es mit Stiftungen großer Vermögensbesitzer aus - die Bill \& Melinda Gates Foundation, zwar noch zu Lebzeiten der Stifter errichtet, erfüllt in einer anderen Größenordnung eine ähnliche Funktion wie die postum errichtete Prunerische Stiftung in Linz: Der Stifter, der für seinen Reichtum bekannt ist, gibt einen Teil seines Vermögens für einen anerkannten Zweck (Innovation in Gesundheit und Wissenschaft) her und betont dies auch im öffentlichen Auftreten. In einer abgemilderten Form war die Statusfrage im 18. Jahrhundert auch bei den häufigen Legaten für religiöse Bruderschaften bedeutsam (viele Testatoren waren Mitglied mehrerer Bruderschaften gewesen). Diese Legate hatten gewöhnlich eine geringe Höhe und erregten gewiss keine besondere Aufmerksamkeit; auffällig wäre es aber gewesen, wenn sie unterblieben wären, und in diesem Sinn waren sie aus Statusgründen sehr wohl notwendig.

Zusammenfassend lässt sich sagen, dass fromme Legate fast durchgehend von einem Eigeninteresse des Erblassers geprägt waren, das entweder transzendent auf die Förderung des Seelenheils oder höchst irdisch auf die Dokumentation des eigenen Status abzielte. Über dieses eigennützige Interesse hinaus bestand aber auch ein eigenständiger Wunsch, Armen und anderen Empfängern milder Gaben etwas Gutes zu tun, einfach weil sie dessen bedürftig waren. In diesem Sinn wohnte den frommen Legaten auch ein Element des Altruismus inne, also einer Haltung, die dazu führt, dass man anderen etwas gibt, ohne davon einen gleichwertigen Nutzen 
zu haben. Außer Betracht bleiben kann der Nutzen, der in der Befriedigung darüber besteht, etwas Gutes getan zu haben. Besonders deutlich wird die Bedeutung des Altruismus im Testieren, wenn Empfänger frommer Legate in Konkurrenz zu Familienangehörigen, Freunden und Bekannten stehen.

\section{Verwandte und Freunde als Nutznießer}

Inwieweit können Legate oder Erbseinsetzungen abseits des frommen und mildtätigen Geschehens als eigennützig oder altruistisch eingeordnet werden? Gewiss nichts Altruistisches liegt in Vermächtnissen an gesetzliche Erben das hinterlassene Vermögen muss irgend jemandem zukommen, und die gesetzlichen Erben sind die dem Erblasser am nächsten stehenden Personen; Vermächtnisse an solche Personen oder ihre Einsetzung als Erben bekräftigen in vielen Fällen lediglich den gesetzlichen $\mathrm{Zu}$ stand (in anderen Fällen dienen sie der Regelung gewisser Abweichungen oder der Präzisierung im Detail). Ob es auch durchgehend eigennützig ist, das Erbrecht wirklich auf die gesetzlichen Erben zu beschränken, sei dahingestellt. In manchen sozialen Zusammenhängen besteht tatsächlich ein Interesse von Erblassern, ihr Vermögen über Generationen hinweg in der Familie zu konzentrieren, wie man etwa an den Fideikommissregelungen im Adel sieht; die Vorstellung einer Unveräußerlichkeit von Vermögenswerten identifiziert das Familieninteresse mit dem Interesse der Familienmitglieder oder jedenfalls des Stifters.

Dass ein solches Familienbewusstsein auch in anderen Milieus bestanden hätte, ist zu bezweifeln. So indizieren die häufigen Besitzwechsel im Zusammenhang mit der Wiederverheiratung verwitweter Grundbesitzer im bäuerlichen Milieu in Westeuropa ein schwaches Bewusstsein von der Wichtigkeit der Erhaltung des Vermö- gens für die leiblichen Nachkommen: Bauer oder Bäuerin verheirateten sich etwa in Österreich oft nach dem Tod des Ehepartners von neuem und übertrugen im Weg der Errichtung einer Gütergemeinschaft die Hälfte des Vermögens an den neuen Gatten. Da sich solches innerhalb weniger Jahre auf beiderlei Seite wiederholen mochte, kam es oft genug dazu, dass Kinder aus früheren Ehen nie an den Grundbesitz gelangten und ein Hof Besitzer erhielt, die mit den Besitzern einer kurz zurückliegenden Zeit gar nicht mehr verwandt waren. ${ }^{7}$ Hätte die Vermögensweitergabe an die leiblichen Nachkommen besondere Wichtigkeit besessen, wären sicherlich andere Regelungen gefunden worden.

Die Einsetzung gesetzlicher Berechtigter als Erben oder Legatare ist also in Hinblick auf den Nutzen für den Erblasser eher unbestimmt, aber jedenfalls kein Akt des Altruismus. Vermächtnisse an andere Empfänger stellen zwar insofern eine Besonderheit dar, als solche Personen $a b$ intestato nichts erhalten würden, doch sind auch solche Vermächtnisse nicht notwendigerweise altruistische Akte. So können etwa Vermächtnisse an Hausbedienstete auch als Leistungen angesehen werden, auf die Bedienstete zwar keinen Rechtsanspruch haben, die aber (etwa nach langer Dienstdauer) so üblich und selbstverständlich waren, dass Bedienstete sie erwarten konnten. In solchen Fällen können sie daher faktisch zu den Kosten von Dienstleuten gerechnet werden, die sich ein Dienstgeber nicht gut ersparen konnte; und die Bezahlung von Dienstleuten ist weder ein eigennütziger noch ein altruistischer Akt.

Auch sonstige Vermächtnisse an Personen außerhalb des Kreises gesetzlicher Erben werden in der Regel nicht ohne weiteres als altruistisch $\mathrm{zu}$ interpretieren sein, wenngleich eine eindeutige Entscheidung meist nicht möglich ist. Meist

\footnotetext{
${ }^{7}$ Vgl. die Fälle in MitTERAUER, Sozialgeschichte der Familie 210-215.
} 
waren solche Legatare mit dem Erblasser persönlich verbunden und das Legat Teil eines Verhältnisses, aus dem beide Seiten materiellen oder immateriellen Nutzen zogen. Nur ausnahmsweise findet man Hinweise darauf, dass ein Legat an Verwandte oder Freunde seinen Grund in der Bedürftigkeit der betreffenden Personen hatte. ${ }^{8}$ Immerhin lässt sich sagen, dass die Selbstverständlichkeit, die man bei Legaten an nahe Angehörige unterstellen kann, bei Legaten an solche außenstehenden Personengruppen fehlt.

\section{Einheitliche Testierprinzipien?}

In welcher Weise standen nun fromme und mildtätige Vermächtnisse mit Vermächtnissen an andere Empfänger in Zusammenhang? Standen Frömmigkeit und Mildtätigkeit mit ihren Besonderheiten - Altruismus, Repräsentationsbedürfnis, Bedacht auf das eigene Seelenheil - in Konkurrenz zu allen anderen Optionen bei der Weitergabe von Vermögen? Oder verlief die Trennlinie eher zwischen den Bedürfnissen der Familie und allen außerhalb der Familie stehenden Personen?

Die empirische Untersuchung stützt sich auf ein Sample von Testamenten des 18. und frühen 19. Jahrhunderts aus Oberösterreich. ${ }^{9}$ Dieses Sample umfasst Personen aus städtischer und ländlicher Umgebung, aus unterschiedlichen Berufsgruppen, Bildungsschichten und Einkommensklassen, und letztwillige Erklärungen unterschiedlicher Art, nämlich eigenhändige, sonstige schriftliche und mündliche Testamente und neben Testamenten auch Kodizille; es bietet

\footnotetext{
${ }^{8}$ Zum Beispiel OÖLA, Alte Statthalterei 1, 169; LGA 897 (Neuhaus), 394.

${ }^{9}$ Es handelt sich um 2828 Testamente des Zeitraums 1700 bis 1820 . $\mathrm{Zu}$ den Quellen und dafür relevanten Fragen PAMMER, Glaubensabfall und Wahre Andacht; DERS., Modeling Religion; DERS., Death and the Transfer of Wealth; DERS., Zeitliche und ewige Dinge.
}

daher die Möglichkeit, verschiedene Faktoren, die auf das Testierverhalten einwirken können, in ihrer verhältnismäßigen Bedeutsamkeit einzuschätzen.

Da im Mittelpunkt dieser Ausführungen nicht der Religiositätswandel vom frühen 18. bis zum frühen 19. Jahrhundert steht, sondern das allgemeine Verhältnis von religiösen zu familiären und sonstigen Motiven bei der Abfassung von letztwilligen Erklärungen, müssen zunächst die spezifischen Auswirkungen des Religiositätswandels auf die Ergebnisse bedacht werden.

Dieser Religiositätswandel bestand in einer breiten Abkehr von der bis zur Mitte des 18. Jahrhunderts herrschenden barocken Frömmigkeit, die in wichtigen Grundzügen beim eingangs besprochenen Testament des Johann Adam Pruner erkennbar geworden ist. Die barocke Frömmigkeit war allgemein gesprochen eine in ihren Ausdrucksformen reich differenzierte und sinnesbetonte Angelegenheit. Für barocke Testamente bedeutet dies, dass in diesen letztwilligen Erklärungen das Bedürfnis erkennbar wird, spirituelle und karitative Anliegen in einem umfangreichen Programm von Legaten für Seelenmessen, Bruderschaften, Gotteshäuser, Spitäler, Siechenhäuser und so weiter zu verwirklichen. Wer wenig zu vererben hatte, teilte auch das Wenige auf mehrere Empfänger auf - möglichst viele Kirchen, Armenhäuser und andere Bedürftige sollten etwas erhalten und so mit der Person und dem Tod des Erblassers konfrontiert werden. Dies korrespondierte mit ausführlichen rhetorischen Erklärungen, die normalerweise in der Art einer Arenga den rechtlich relevanten Punkten vorangestellt wurden und in formelhafter Weise Leben, Sterben, Tod, Gott, Heilige und den Glauben thematisieren.

In der zweiten Hälfte des 18. Jahrhunderts vollzog sich nach und nach ein Abschied von dieser Art des Testierens. In den siebziger und achtziger Jahren wurde bereits deutlich erkennbar, dass das barocke Muster nicht mehr verbindlich war, und die folgenden Jahrzehnte bis in die 
Restaurationszeit hinein unterstrichen dies. Der Veränderungsprozess erfolgte nicht einheitlich: Ein Teil der Testatoren jener Zeit folgte den Prinzipien des Reformkatholizismus, die im Denken der Zeit und seiner Umsetzung in Verwaltung und Politik dominant wurden. Im Reformkatholizismus verschoben sich die Gewichte von Frömmigkeitsinhalten und -formen, die als unwesentlich angesehen wurden (etwa von der Heiligenverehrung oder auch sinnesbetonten, stark rituell bestimmten oder magisch anmutenden Handlungen), zu den als zentral eingeschätzten Dingen (zur Konzentration der Andacht auf Gott, Betonung verinnerlichter Gläubigkeit, zum Nutzen der Religion für die äußeren Lebensbedingungen der Menschen); Religion erschien also weiterhin als unverzichtbar, sie sollte aber anders praktiziert werden. Ein anderer Teil der Testatoren bietet hingegen das Bild einer sich säkularisierenden Gesellschaft: Ihre letztwilligen Erklärungen lassen jeden Bezug auf Religion vermissen, der eigene Tod war kein Anlass für spirituelle Überlegungen oder Vorsorge für die eigene Seele mehr.

Insgesamt gingen auf diese Weise binnen weniger Jahrzehnte die Summen, die in den Testamenten für fromme und mildtätige Zwecke gewidmet wurden, auf einen Bruchteil früherer Werte zurück. Dieser Vorgang war keine Besonderheit irgendeines Landes; er lässt sich in Frankreich ebenso beobachten wie in rheinischen und westfälischen Städten oder eben auch in Österreich. Für diesen Rückgang wurden verschiedene Erklärungen vorgebracht. Eine naheliegende Erklärung sieht den Grund in der großen geistesgeschichtlichen Bewegung der Zeit: Die Aufklärung erfasste die christlichen Konfessionen, allgemein in Form einer Abkehr Vieler von der Religiosität der Barockzeit und im besonderen in der Öffnung verschiedener neuer Wege von aufgeklärter oder reformerisch gesinnter Religiosität bis hin zur Abkehr von christlicher Spiritualität schlechthin.

\section{Empirischer Befund}

Vor dem Hintergrund der allgemeinen geistesgeschichtlichen Entwicklung soll nun zunächst das Testierverhalten in seinen verschiedenen Möglichkeiten erklärt werden. Es ist anzunehmen, dass es gewisse mit den aktuellen Lebensund Zeitumständen verbundene Faktoren gab, die im Einzelfall bestimmten, wen Testatoren als Erben einsetzten und wem sie Legate zudachten. Erst wenn man das Gewicht dieser Faktoren berücksichtigt und feststellt, welcher Teil des Verhaltens durch sie nicht erklärt werden kann, kann man sagen, ob es übergreifende Muster in der Zuwendung von Vermögen an verschiedene Empfängergruppen gibt.

Als mögliche Empfänger testamentarischer Zuwendungen kommen folgende Gruppen in Frage:

- Verwandte: Ehepartner, Kinder, sonstige Verwandte;

- Bekannte: Patenkinder, Bedienstete, sonstige nicht verwandte Personen;

- Empfänger frommer Legate: Bruderschaften, Seelenmessen, Gotteshäuser, Armeninstitutionen

Für jede dieser Gruppen von Empfängern ist zu untersuchen, welche Faktoren in welchem Ausmaß bestimmten, ob sie testamentarisch bedacht wurden, und was die Höhe dieser Zuwendungen bestimmte. Bestimmende Faktoren dieser Art sind:

- Form des Testaments: eigenhändige, sonstige schriftliche, mündliche Testamente;

- Umstände der Testamentserrichtung: Anwesenheit bestimmter Personengruppen als Zeugen oder aus anderen Gründen, etwa Priester, Notare, Anwälte, Ärzte;

- Familienstand des Erblassers

- Zahl der Kinder

- Soziale Position: Beruf, Bildung, Einkommen, Vermögen;

- Adeliger Status 
- Jahr der Testamentserrichtung

Für jede Gruppe von Empfängern wurde der Einfluss dieser Faktoren auf Vorhandensein und Ausmaß von entsprechenden Vermächtnissen und Erbseinsetzungen geschätzt. Das heißt es wurde etwa untersucht, wie sich Beruf, Status, Jahr und so weiter darauf auswirkten, ob jemand seine Kinder zu Universalerben einsetzte oder ihnen Legate vermachte; ebenso für Ehepartner, verschiedene sonstige Personengruppen und die frommen Legate. Dazu wurden statistische Standardverfahren verwendet, die es erlauben, nichtlineare Einflüsse dieser Art zu bestimmen. In die Analyse wurden nur Erblasser einbezogen, die entweder verheiratet oder verwitwet waren, da nur diese Personen legitime Kinder hinterließen. Die Ergebnisse dieser Untersuchungen sind im bereits publizierten Material dokumentiert, ${ }^{10}$ einige charakteristische Einzelheiten seien hier hervorgehoben.

Offenkundig ist die unterschiedliche Bewertung der Familie je nach sozialem Zusammenhang. Dies betraf sowohl die engere Familie als auch entferntere Verwandte. Testatoren standen vor der Entscheidung, ihre Ehegatten, ihre Kinder oder ihre entfernteren Verwandten exklusiv oder miteinander als Erben einzusetzen. Deutliche Unterschiede ergaben sich dabei vor allem zwischen adeligen Erblassern und dem Rest der Bevölkerung: Adelige neigten dazu, nicht ihre Ehegatten, sondern ihre Kinder als Erben einzusetzen, offensichtlich ein Ergebnis der besonderen Wichtigkeit, die man im Adel blutsverwandtschaftlichen Bindungen zuschrieb. Ein Erbrecht von Ehegatten hätte dazu geführt, dass das Vermögen in weiterer Folge in eine andere Familie gelangen konnte, da sich viele verwitwete Personen neuerlich verheirateten, ein Vorgang, den es, wie beschrieben, im bäuerlichen Milieu oft gab; bei einer Wiederverheiratung mochten Vermögenswerte auf den neuen Gatten

${ }^{10}$ PAMMER, Death and the Transfer of Wealth. übertragen und von diesem an seine Kinder vererbt werden. Die Einsetzung der eigenen Kinder als Erben und die Beschränkung des Ehegatten auf ein Legat verhinderte dies; zusätzlich zu den in einer Reihe von Familien ohnehin bestehenden Fideikommissregelungen wurde also auch beim Allodialvermögen darauf geachtet, die Verwandten in gerader Linie $\mathrm{zu}$ bevorzugen, wobei beim Allodialvermögen oft auch Kinder bedacht wurden, die in der Fideikommissnachfolge benachteiligt waren. In der sonstigen Bevölkerung war der Anteil von Ehegatten, die man als Erben einsetzte, deutlich höher.

Im bäuerlichen Bereich trat dazu noch die Besonderheit, dass verhältnismäßig oft auch entferntere Verwandte als Universalerben eingesetzt wurden. Dies hatte seine Ursache in der in Österreich herrschenden Flexibilität bei der Übergabe von landwirtschaftlichen Betrieben: Die Betriebe, insbesondere die dazugehörigen Immobilien, repräsentierten zwar einen beträchtlichen Vermögenswert, doch war man leicht bereit, das Eigentum daran schon zu Lebzeiten allenfalls auch an außenstehende Personen zu übertragen. Eine solche Betriebsübergabe war unter anderem mit regelmäßigen leibrentenartigen Zahlungen der Betriebsübernehmer an die abtretenden Eigentümer verbunden. Selbstverständlich kam es nicht selten vor, dass Bauern in einem Alter starben, in dem sie ihren Betrieb noch nicht übergeben hatten; in solchen Fällen wurden die neuen Eigentümer der Betriebe testamentarisch in ähnlicher Weise bestimmt, wie es bei Übergaben zu Lebzeiten üblich war, das heißt man entschied sich ganz pragmatisch für die geeignetste Person: Wenn leibliche Kinder vorhanden waren, die das erforderliche Alter besaßen, erhielten diese den Hof; wenn die Kinder zu jung waren, kam der überlebende Ehegatte zum Zug; wenn keine Kinder vorhanden waren und der Ehegatte, etwa aus Altersgründen, den Hof nicht weiterführen wollte, entschied man sich für Außenste- 
hende, etwa entferntere Verwandte oder durchaus auch nicht verwandte Personen.

Eine besondere Gruppe von nicht verwandten Personen waren Bedienstete von Angehörigen der oberen Klassen, insbesondere von Adeligen, aber auch von Angehörigen von Bildungsberufen. Solche Dienstgeber neigten dazu, ihren Bediensteten teils großzügige Vermächtnisse zu machen (als Universalerben kamen diese Bediensteten freilich nicht in Frage). Dies hatte seinen Grund in der großen persönlichen Nähe, zumal solche Bedienstete im Haushalt oft in der persönlichen Betreuung ihrer Dienstgeber tätig waren. Im Gegensatz dazu erhielten bäuerliche Dienstboten von ihren Dienstgebern normalerweise keine testamentarischen Zuwendungen, da bäuerliche Dienstboten mit wenigen Ausnahmen dem Betrieb und nicht der persönlichen Betreuung des Bauern dienten und verhältnismäßig oft wechselten. Auch sonstige nicht verwandte Personen wurden von Adeligen und anderen Angehörigen der oberen Klassen häufiger bedacht als von bäuerlichen Erblassern.

Häufigkeit und Ausmaß frommer Legate variierten in vielfältiger Weise entsprechend den Randbedingungen. Angehörige der Unterschicht neigten verhältnismäßig wenig dazu, solche Legate zu verfügen, ebenso Bauern. Verglichen mit diesen beiden Gruppen, findet man fromme Legate bei Handwerkern und Beamten deutlich häufiger. Auch Angehörige von Bildungsberufen oder des Adels machten öfter solche Legate. Bezieht man unverheiratete Personen in die Analyse mit ein, zeigt sich ein $\mathrm{Zu}$ sammenhang mit dem Familienstand: Unverheiratete gaben eher etwas für fromme Zwecke, am deutlichsten zeigt sich dies bei Priestern. Alle diese Ergebnisse gelten unabhängig vom Wert der Vermächtnisse, das heißt nur für die Frage, ob überhaupt fromme Legate in einem Testament vorkamen. Insofern sind die Ergebnisse nicht trivial, da auch Unterschichtangehörige geringfügige Legate für fromme Zwecke hätten machen können. Berücksichtigt man auch den
Wert der Legate, zeigt sich erwartungsgemäß, dass in den vermögenderen Klassen, das heißt etwa unter Angehörigen von Bildungsberufen oder des Adels, größere Beträge für fromme Zwecke vorgesehen waren. Geringer wurde der Wert frommer Vermächtnisse mit der Anzahl der Kinder, die ein Erblasser hatte.

Formale Merkmale der Testamente, die mit dem Ablauf der Testamentserrichtung und gewissen äußeren Umständen assoziiert sind, stehen ebenfalls in Zusammenhang mit dem Testierverhalten. Manche Zusammenhänge überraschen wenig. So findet man in mündlichen Testamenten öfter Ehegatten als Universalerben; dies ist sicherlich ein Ergebnis des Umstands, dass Ehegatten häufig beim mündlichen Testament anwesend waren. Weniger trivial ist der Zusammenhang zwischen der eigenhändigen Errichtung eines Testaments und dem Vorhandensein und Ausmaß von frommen Vermächtnissen: Personen, die ihr Testament mit eigener Hand schrieben, sahen unter sonst gleichen Bedingungen im Durchschnitt häufiger fromme Legate vor und widmeten diesem Zweck auch größere Beträge. Dies ist deshalb wichtig, weil eigenhändige Testamente in der Regel längere Zeit vor dem Tod errichtet wurden (der Abstand zwischen dem Datum des Testaments und dem Todeszeitpunkt war bei diesen letztwilligen Erklärungen größer als bei anderen schriftlichen Testamenten und viel größer als bei mündlichen Erklärungen, die oft wirklich erst in letzter Minute abgegeben wurden). Das heißt, Testatoren, die ihr Testament selbst schrieben, hatten viel Zeit zur Überlegung und waren im Allgemeinen noch bei besserer Gesundheit. Es kann also keine Rede davon sein, dass fromme Legate überwiegend ein Ergebnis der Einflussnahme durch Notare, Anwälte oder Priester gewesen wären, wenngleich etwa notarielle Testamente ebenfalls eine stärkere Tendenz zu frommen Vermächtnissen (aber auch zu Vermächtnissen an sonstige familienfremde Empfänger) zeigen. 
Ganz wesentlich war für die frommen Legate schließlich der Zeitfaktor, das heißt die Frage, ob ein Testament noch in die Zeit der barocken Frömmigkeit, in die Übergangszeit der siebziger, achtziger und neunziger Jahre des 18. Jahrhunderts oder in die Zeit ab den neunziger Jahren fiel. Fromme Legate wurden in der Übergangszeit seltener und nahmen dem Wert nach $a b$. Eine solche Entwicklung gab es für keine andere Gruppe von Legataren und Erben; zum Teil gab es hier gar keine signifikante Veränderung im Zeitablauf, zum Teil erfolgte eine Verschiebung in der Weise, dass man bestimmten Personengruppen, die früher als Universalerben in Frage gekommen wären, im Lauf der Zeit lieber ein Legat zudachte, oder umgekehrt.

Alle diese Befunde erklären einen mehr oder weniger großen Anteil am gesamten beobachteten Geschehen. Da die verwendeten Daten Individualdaten sind, also Daten, die sich auf Einzelpersonen und ihre Testamente beziehen, ist normalerweise mehr als die Hälfte der beobachteten Unterschiede zwischen den Personen mit den verwendeten Modellen nicht zu erklären. Das liegt einerseits daran, dass in historischen Untersuchungen wichtige erklärende Daten oft nicht verfügbar sind, andererseits und zum gröBeren Teil daran, dass sich Menschen im Einzelfall oft in nicht vorhersagbarer Weise verhalten, da eben vieles am menschlichen Verhalten der spontanen persönlichen Entscheidung unterliegt.

Es lässt sich also ein gewisser Teil des Testierverhaltens durch die genannten Faktoren erklären, doch verbleibt ein Rest, der dadurch eben nicht zu erklären ist. Dieser Rest ist somit unabhängig von den diskutierten Randbedingungen wie Vermögen, Beruf, Familiensituation und zeittypischen Umständen der geistesgeschichtlichen Entwicklung. War dieser nicht erklärbare Teil des Testierverhaltens vom völligen Zufall bestimmt, oder war es in dem Sinn konsistent, dass Testatoren hinsichtlich der potentiellen Empfänger ihrer Vermögenswerte im Großen und Ganzen eine ähnliche Linie verfolgten? Gab es spezifische Koinzidenzen zwischen Zuwendungen an die eine Gruppe von Empfängern und Zuwendungen an eine andere? Mögliche Varianten wären:

- Konkurrenz innerhalb der Familie: Testatoren konnten bei der Entscheidung zwischen Ehegatten und Kindern beide Seiten gleichmäßig bedenken (also Zuwendungen an Ehegatten korrelieren positiv mit Zuwendungen an Kinder) oder die eine Seite zugunsten der anderen Seite ausschließen (die Zuwendungen an Ehegatten und Kinder korrelieren negativ miteinander) oder in dieser Hinsicht kein einheitliches Verhalten zeigen (die Zuwendungen an Ehegatten und Kinder korrelieren nicht miteinander).

- Konkurrenz zwischen Familie und Außenstehenden: Testatoren konnten zwischen der Familie und Außenstehenden entscheiden, indem sie entweder an alle gaben (positive Korrelation) oder an die eine Seite gaben und die andere dabei ausschlossen (negative Korrelation) oder uneinheitlich agierten (keine Korrelation).

- Konkurrenz zwischen frommen Legataren und sonstigen familienfremden Empfängern: Testatoren konnten unter den potentiellen Empfängern außerhalb der Familie wählen, indem sie dann, wenn sie fromme Legate bestimmten, auch entfernte Verwandte, Freunde oder Bedienstete bedachten (positive Korrelation), indem sie einen spezifischen Empfängerkreis bedachten und den Rest ausschlossen (negative Korrelation) oder sich uneinheitlich verhielten (keine Korrelation).

Alle diese möglichen Zusammenhänge gelten ohne Auswirkung der oben bereits berücksichtigten sozialen Faktoren.

\section{Tabellen 1 und 2}

Die Ergebnisse (Tabellen 1 und 2) lassen vermuten, dass die Testatoren im Allgemeinen typischen Mustern folgten. Im Fall des ersten oben 
genannten Problems, nämlich der Entscheidung, wie man Ehegatten im Vergleich insbesondere zu den Kindern behandeln sollte, wird deutlich, dass Ehegatten und Kinder einander als Erben meist ausschlossen. Abweichend von der gesetzlichen Erbfolge, die eine Teilung des Erbes zwischen Ehegatten und Kindern vorsah, entschieden sich die Testatoren tendenziell für eine Beschränkung des Erbrechts entweder auf die Kinder oder auf den Gatten - es gibt einen signifikant negativen Zusammenhang zwischen der Ernennung von Kindern und der Ernennung von Gatten zu Universalerben. Man ernannte also nicht Kinder und Gatten miteinander zu Erben, und das Ergebnis schwankte auch nicht von Fall zu Fall in beliebiger Weise, sondern die Testamente dienten hier wirklich in der Regel der Entscheidung innerhalb der Familie. Hingegen gab es hinsichtlich der Legate an Kinder und Ehegatten kein solches durchgehend bestimmendes Muster: Ein Teil der Testatoren bedachte Ehegatten und Kinder mit Legaten, ein anderer Teil gab den einen etwas und ließ die anderen leer ausgehen - ein klarer Zusammenhang bestand nicht.

Das zweite Problem, die Konkurrenz zwischen der engeren Familie und außenstehenden Empfängern, wurde hinsichtlich der Erbteilsrechte einigermaßen klar entschieden: Wenn Mitglieder der engeren Familie, insbesondere der Ehegatte, einen Erbteil erhielten, war damit die weitere Verwandtschaft und Freundschaft aus dem Spiel. Anders sieht es mit Vermächtnissen aus: Vermächtnisse an Kinder und Ehegatten schlossen Vermächtnisse an außenstehende Personen nicht aus, erforderten sie aber auch nicht; hier gibt es also keinen signifikanten Zusammenhang.

Die dritte Frage galt der Auswahl unter der Vielzahl möglicher Erben und Legatare außerhalb der engeren Familie. Sollte man gezielt bestimmte Empfänger bedenken und andere damit auslassen? Oder wollte man breit streuen? Die Ergebnisse zeigen, dass bei der Ernennung von Universalerben ein Ausschlussprinzip herrschte: Wer Verwandten ein Erbteil gab, gab nicht auch noch den sonstigen Freunden, und umgekehrt (allerdings wurden Empfänger auBerhalb der engeren Familie überhaupt selten zu Universalerben). Anders sieht es bei den Vermächtnissen aus, da es offenbar üblich war, entweder allen Empfängern außerhalb der engeren Familie etwas zu geben oder niemandem: Wer fromme Legate machte, gab meist auch etwas an Verwandte, Freunde, Patenkinder und so weiter; wer an Verwandte gab, von dem durften sich auch Bedienstete und Freunde etwas erhoffen.

\section{Ergebnisse und Zusammenfassung}

Das Ergebnis gibt ein verhältnismäßig einfaches und klares Bild, soweit es in einem Testament um die Einsetzung von Erben ging. Diese Einsetzung war in der Regel exklusiv auf eine Person (oder mehrere Personen aus derselben Gruppe, etwa mehrere Kinder) beschränkt und schloss damit den Rest aus. Dies ist an sich naheliegend, da viele Testamente sicherlich gerade wegen der Erbseinsetzung gemacht wurden, wäre aber grundsätzlich nicht die einzige Möglichkeit, da man ja mehrere Erben nebeneinander hätte ernennen können. Relevant ist dieser Punkt vorrangig bei der Verteilung innerhalb der Familien, da die Erben zum größten Teil Ehepartner oder Kinder waren.

Wesentlich komplexer ist die Situation bei den Vermächtnissen. Als Legatare kamen auch Empfänger außerhalb der Familie in Frage, und diese wurden auch wirklich oft bedacht. Gab es hier einen ausschließenden Gegensatz zwischen Familie und außenstehenden Empfängergruppen, etwa in der Art, dass Testatoren, die Familienangehörige bedachten, Außenstehende ausschlossen, und umgekehrt? Diese Frage kann man verneinen: Familie einerseits und Freunde, 


\begin{tabular}{|c|c|c|c|c|}
\hline & Ehegatten & Kinder & Verwandte & Freunde \\
\hline Kinder & $\begin{array}{l}-0,1218^{* * * *} \\
(0,0235)\end{array}$ & & & \\
\hline Verwandte & $\begin{array}{l}-0,1111^{* * * *} \\
(0,0238)\end{array}$ & $\begin{array}{l}-0,0323 \\
(0,0218)\end{array}$ & & \\
\hline Freunde & $\begin{array}{l}-0,0995^{* *} \\
(0,0306)\end{array}$ & $\begin{array}{l}-0,0417 \\
(0,0280)\end{array}$ & $\begin{array}{l}-0,1574^{* * *} \\
(0,0274)\end{array}$ & \\
\hline Fromme Empfänger & $\begin{array}{l}-0,0486 \\
(0,0559)\end{array}$ & $\begin{array}{l}0,0114 \\
(0,0510)\end{array}$ & $\begin{array}{l}-0,1949^{* *} \\
(0,0502)\end{array}$ & $\begin{array}{l}-0,0118 \\
(0,0392)\end{array}$ \\
\hline \multicolumn{5}{|c|}{ 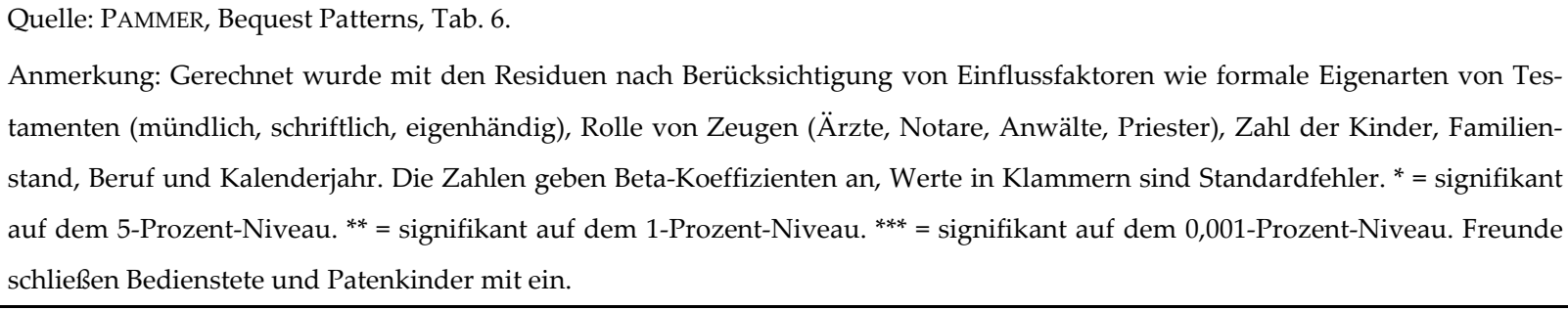 } \\
\hline
\end{tabular}

Bekannte und Empfänger milder Gaben andererseits schlossen einander nicht aus, sondern sind überhaupt nicht miteinander korreliert.

Dieses Ergebnis ist deshalb relevant, weil es eine Frage erhellt, die im Zusammenhang mit dem Religiositätswandel im späten 18. Jahrhundert aufgeworfen wurde. Philippe Ariès führte das Zurückgehen und teilweise Verschwinden der frommen Legate in den Testamenten jener Zeit nämlich nicht auf einen wirklichen Wandel der Religiosität, sondern lediglich auf einen Wandel in Testiergewohnheiten zurück: Die Funktion von Testamenten habe sich in der zweiten Hälfte des 18. Jahrhunderts im Zusammenhang mit der Veränderung innerfamiliärer Beziehungen gewandelt. ${ }^{11}$ Die gefühlsmäßigen Bindungen innerhalb der Familie hätten sich gegenüber der vorangegangenen Zeit verstärkt, was dazu geführt habe, dass man für dahingegangene Angehörige und ihr Seelenheil aus Liebe zu ihnen von sich aus tätig geworden sei. Seelenmessen zugunsten des Verstorbenen, mildtätige Hand-

${ }^{11}$ ARIÈS, Geschichte des Todes 596-599. lungen in seinem Namen hätten die Überlebenden nun jedenfalls veranlasst, auch ohne durch testamentarische Vorsorge dazu verhalten $\mathrm{zu}$ sein. Daher hätten immer mehr Erblasser auf solche Klauseln verzichtet, weil sie sich auf ihre Familie verlassen konnten. Doch würde dies nicht bedeuten, dass man den Wert von frommen Handlungen geringer geschätzt hätte als früher; von einer Veränderung der Religiosität könne daher nicht gesprochen werden.

Gegen diese These sprechen verschiedene Befunde. So wurden religiöse Bekundungen in Testamenten überhaupt seltener, auch die rhetorischen Aussagen über Glaubensangelegenheiten, die mit dem Seelenheil und der Affektivität in den Familien nichts zu tun hatten. Auch wurde im Lauf der Zeit nicht nur die Häufigkeit frommer Legate geringer, sondern auch der Wert solcher Legate; dieser Wert sank auch mit der Zahl der Kinder, die ein Erblasser hatte. Hätten die Erblasser darauf vertraut, dass ihre Angehörigen ohnehin aus kindlicher Liebe alles für ihre arme Seele tun würden, hätten sie wohl 


\begin{tabular}{|c|c|c|c|c|c|c|}
\hline & Ehegatten & Kinder & Verwandte & Patenkinder & Bedienstete & Freunde \\
\hline Kinder & $\begin{array}{l}0,0298 \\
(0,0176)\end{array}$ & & & & & \\
\hline Verwandte & $\begin{array}{l}0,0184 \\
(0,0171)\end{array}$ & $\begin{array}{l}-0,0243 \\
(0,0209)\end{array}$ & & & & \\
\hline Patenkinder & $\begin{array}{l}-0,0509 \\
(0,0305)\end{array}$ & $\begin{array}{l}0,009 \\
(0,0374)\end{array}$ & $\begin{array}{l}0,2844^{* * *} \\
(0,0380)\end{array}$ & & & \\
\hline Bedienstete & $\begin{array}{l}0,0137 \\
(0,0194)\end{array}$ & $\begin{array}{l}0,0315 \\
(0,0237)\end{array}$ & $\begin{array}{l}0,1358^{* * *} \\
(0,0243)\end{array}$ & $\begin{array}{l}0,0991^{* * *} \\
(0,0135)\end{array}$ & & \\
\hline Freunde & $\begin{array}{l}0,0065 \\
(0,0193)\end{array}$ & $\begin{array}{l}0,021 \\
(0,0236)\end{array}$ & $\begin{array}{l}0,1881^{\text {*** }} \\
(0,0239)\end{array}$ & $\begin{array}{l}0,1109^{* * *} \\
(0,0134)\end{array}$ & $\begin{array}{l}0,3019^{* * *} \\
(0,0204)\end{array}$ & \\
\hline $\begin{array}{l}\text { Fromme Emp- } \\
\text { fänger }\end{array}$ & $\begin{array}{l}0,0219 \\
(0,0150)\end{array}$ & $\begin{array}{l}0,0854^{\text {*** }} \\
(0,0183)\end{array}$ & $\begin{array}{l}0,1245^{* * *} \\
(0,0187)\end{array}$ & $\begin{array}{l}0,0223^{*} \\
(0,0106)\end{array}$ & $\begin{array}{l}0,0480^{* *} \\
(0,0166)\end{array}$ & $\begin{array}{l}0,0821^{\text {*** }} \\
(0,0167)\end{array}$ \\
\hline \multicolumn{7}{|c|}{$\begin{array}{l}\text { Quelle: PAMMER, Bequest Patterns, Tab. } 7 . \\
\text { Anmerkung: Gerechnet wurde mit den Residuen nach Berücksichtigung von Einflussfaktoren wie formale Eigenarten von Testa- } \\
\text { menten (mündlich, schriftlich, eigenhändig), Rolle von Zeugen (Ärzte, Notare, Anwälte, Priester), Zahl der Kinder, Familienstand, } \\
\text { Beruf und Kalenderjahr. Die Zahlen geben Beta-Koeffizienten an, Werte in Klammern sind Standardfehler. }{ }^{*}=\text { signifikant auf dem } \\
\text { 5-Prozent-Niveau. }{ }^{* *}=\text { signifikant auf dem 1-Prozent-Niveau. }{ }^{* *}=\text { signifikant auf dem 0,001-Prozent-Niveau. }\end{array}$} \\
\hline
\end{tabular}

nicht im Lauf der Zeit geringere Beträge für diesen Zweck gewidmet, sondern gar nichts darüber bestimmt. Größere Wahrscheinlichkeit hat die Annahme, dass Testatoren im Lauf der Zeit diesen Dingen einen immer geringeren Wert beimaßen und dass jene Erblasser, die mehr Kinder hatten, bei Ausgaben, die das für die Familie verbleibende Vermögen verringerten, sparsamer wurden.

Die Geschichte frommer Legate ist also wohl keine Familiengeschichte im Sinn der These von Ariès. Aber wie ist es dann zu erklären, dass Testatoren, die fromme Vermächtnisse machten, auch dazu neigten, verschiedenen anderen Empfängern außerhalb ihrer Familie etwas zukommen zu lassen? Es ist dies ein Verhalten, das man als „Außenorientierung“ bezeichnen könnte. Es steht nicht in einem Gegensatz zur Orientierung an den Bedürfnissen der Familie, weil diese Zuwendungen an Außenstehende mit Zu- wendungen an die engere Familie eben nicht korreliert sind; das heißt, dass manche Testatoren Zuwendungen an die Familie machten, andere an Außenstehende, wieder andere an beide Personengruppen. Es ist also ein unabhängiges Geschehen. Wesentlich daran ist, dass jene, die überhaupt etwas an Außenstehende gaben, dabei gleich eine ganze Reihe potentieller Empfänger berücksichtigten.

Familienorientierung und Außenorientierung schlossen einander also nicht aus, gingen aber auch nicht notwendigerweise Hand in Hand. Der Außenorientierung wohnte fraglos ein Element des Altruismus inne, sie erschöpft sich aber nicht darin. Das fromme Legat war selbst von verschiedenen Zwecken getragen, vom Eigeninteresse der Testatoren ebenso wie von der Sorge um unbekannte Bedürftige. Diese Bereitschaft, Menschen außerhalb der Familie etwas zukommen zu lassen, war aber offenbar 
nur ein Aspekt einer Disposition, die auch zu Vermächtnissen an entfernte Verwandte, Freunde und sonstige Bekannte veranlasste.

\section{Literatur:}

Philippe ARIÈs, Geschichte des Todes (München 1982).

Jacques LE GOFF, Die Geburt des Fegefeuers (Stuttgart 1984).

Michael MITTERAUER, Sozialgeschichte der Familie als landeskundlicher Forschungsgegenstand. Auswertungsmöglichkeiten historischer Personenstandslisten, in: DERS., Historisch-anthropologische Familienforschung. Fragestellungen und Zugangsweisen (Wien-Köln 1990) 191-231.

Michael PAMMER, Glaubensabfall und Wahre Andacht. Barockreligiosität, Reformkatholizismus und Laizismus in Oberösterreich 1700-1820, (Wien-München 1994).

Michael PAMMER, Modeling Religion. Bureaucratic Reform and the Transformation of Popular Piety in the 18th Century, in: Historical Social Research 19/4 (1994) 4-25.

Michael PAMMER, Death and the Transfer of Wealth. Bequest Patterns and Cultural Change in the
Eighteenth Century, in: Journal of Social History 33/4 (2000) 913-934.

Michael PAMMER, Testamente und Verlassenschaftsabhandlungen (18. Jahrhundert), in: Josef PAUSER, Martin Scheutz, Thomas Winkelbauer (Hgg.), Quellenkunde der Habsburgermonarchie (16.-18. Jahrhundert). Ein exemplarisches Handbuch (= MIÖG Erg.Bd.44,Wien-München 2004) 495510.

Michael PAMMER, Zeitliche und ewige Dinge: Hinterlassenschaften und Seelenheil in oberösterreichischen Testamenten des 18. Jahrhunderts, in: Markwart Herzog, Cecilie Hollberg (Hgg.), Seelenheil und irdischer Besitz. Testamente als Quellen für den Umgang mit den "letzten Dingen“ (Konstanz 2007) 79-84.

Sammlung der Kaiserlich=Königlichen Landesfürstlichen Gesetze und Verordnungen in PublicoEcclesiasticis vom Jahre 1767 bis Ende 1782 (Wien 1782).

\section{Abkürzungen:}

LGA Landesgerichtsarchiv

Siehe auch das allgemeine Abkürzungsverzeichnis: http://www.rechtsgeschichte.at/beitraege/abk.pdf 